\title{
COMPARISON OF AUTOMATIC STARRSED RS ERYTHROCYTE SEDIMENTATION RATE WITH MANUAL WESTERGREN METHOD
}

\author{
Ninik Sukartini, Nuri Dyah Indrasari, Siskawati Suparmin \\ Department of Clinical Pathology, Faculty of Medicine, Indonesia University/Dr. Cipto Mangunkusumo Hospital Jakarta, Indonesia. E-mail: \\ siskawati.suparmin@gmail.com
}

\begin{abstract}
This study aimed to compare the results of the Erythrocyte Sedimentation Rate (ESR) measurements using Starrsed RS (30- and 60-minute mode) and manual Westergren method. The study also investigated the effect of high leukocytes and hematocrit $(\mathrm{Ht})$ on Starrsed RS ESR measurements. A cross-sectional study using K3EDTA anticoagulated blood samples from 140 subjects was conducted. A total of 21 samples with leukocyte counts $>50000 / \mu \mathrm{L}$ was examined. The effect of Ht was studied using data from comparative study subjects. The 60-minute mode Starrsed RS correlated very strongly $[r=0.98(95 \%$ CI 0.97 to 0.98 ), $p<0.0001$ ], had a good agreement [mean bias $-0.4 \mathrm{~mm}$ ( $95 \%$ CI -1.46 to 0.74 )], and showed no significant different results with manual Westergren method. The 30-minute mode Starrsed RS also had a solid correlation $[r=0.97$ (95\%CI 0.96 to 0.98$), p<0.0001$ ] and a good agreement [mean bias-2.9 mm (95\% CI -4.23 to -1.58$)$ ], but had a systematic difference with manual Westergren method. There was a significant difference between the manual method and Starrsed RS in samples with high leukocytes $(p=0.0208)$. There were no significant differences of ESR results measured by Starrsed RS and manual Westergren method in the group of $\mathrm{Ht} \leq 35 \%$ and $>35 \%$. The ESR on the Starrsed RS should still be read at 60 minutes. Leukocytes $>50$ 000/ $\mu \mathrm{L}$ may affect ESR measurements on Starrsed RS but no effect of hematocrit was observed.
\end{abstract}

Key words: Automated, erythrocyte sedimentation rate, Starrsed, Westergren

\section{INTRODUCTION}

Erythrocyte Sedimentation Rate (ESR) is a general laboratory test to assess acute phase proteins in chronic diseases. ${ }^{1}$ Despite its limitation and other specific inflammatory markers that have been found, ESR is still widely used for screening and monitoring of infectious diseases, autoimmune, malignancies, and other diseases affecting plasma proteins as it is a relatively inexpensive and straightforward test. ${ }^{2}$

The Westergren method is defined by the Clinical and Laboratory Standards Institute (CLSI) as the gold standard for ESR measurement. ${ }^{3}$ The use of conventional Westergren method needs a large volume of blood and 60-minutes of time. Several methods based on conventional methods are developed to overcome these limitations. The ESR procedure is unable to be calibrated, thus it is vulnerable to various error sources. There is no method for ensuring that ESR measurement is not affected by multiple factors, such as hematocrit, erythrocyte size, morphology, and other confounding factors. ${ }^{4}$ Since 1991, some evaluations had been made for ESR by using alternative methods. However, most of these evaluations failed to calibrate their measurements according to the ICSH guidelines, which resulted in decreased standardization and comparability of the ESR. ${ }^{1}$

Starrsed RS from RR Mechatronics is an automated ESR measuring instrument using the Westergren reference method. ${ }^{3}$ Based on the previous study, it was found that ESR reading of 60 minutes could be predicted using a compensation table of ESR 30 minutes (correlation coefficient $=0.984$ ). Both methods had a great correlation. ${ }^{5}$

This study aimed to compare ESR measurement results using Starrsed RS automated instrument (30 minute and 60-minute mode) to the Westergren manual method. Also, this study also observed the influence of high leukocytes and hematocrit against ESR measurement using Starrsed RS.

\section{METHODS}

This study was conducted at the Central Laboratory of Clinical Pathology Department, Cipto Mangunkusumo National Center General Hospital (RSCM), Faculty of Medicine, University of Indonesia Jakarta, from February to March 2017. Research subjects were recruited from all patients who did a 
complete hematological examination. Venous blood sample for the comparative test was collected into a K3EDTA tube. The tube should be flipped back at least eight times. Two tubes of blood were collected for each subject. Blood in the first tube was used for complete hematological examination, including ESR measurement by manual Westergren method. The $0.9 \% \mathrm{NaCl}$ was used to dilute the patient's blood in the ESR manual method with a ratio of blood to $0.9 \%$ $\mathrm{NaCl}$ of $4: 1{ }^{6}$ Blood in the second tube was used for ESR measurement by Starrsed RS (60-minute and 30-minute modes).

Starrsed RS is an automated ESR measuring instrument using the Westergren reference method principle. A sample of blood in an EDTA tube was placed on a Starrsed rack unit before it was tested. The barcode reader in the instrument read the barcode on the sample tube. Then, the instrument started taking samples from the tube to fill the pipette. Samples were automatically diluted in the instrument with a diluent containing $3.2 \%$ sodium citrate with a ratio of $4: 1$. Starrsed RS used standard Westergren glass pipette. The ESR was read at 60th minutes or $30^{\text {th }}$ minutes, depending on the chosen mode. There were two programs for ESR measurement on Starrsed RS instrument, 60-minute mode (as recommended by the manufacturer) and 30-minute mode. The automated reading of the Westergren sedimentation pipette was carried out by moving an optical sensor along with the pipettes. While the sensor was moving, a reading was made every $0.25 \mathrm{~mm}$. The sensor was reading the absorption of infrared light through the Westergren pipette filled with blood. From these readings, value at the number of absorption levels was determined. All absorption figures were relative to the darkest and lightest reading (darkest $=100 \%$ and the lightest $=0 \%$ ). By definition, the levels were $87.5 \%$ for cells/plasma separation, $75 \%$ for hazy detection, and $50 \%$ for meniscus detection). For a 30-minute mode test, the results were corrected using a software program in the instrument, and it was a prediction of ESR at $60^{\text {th }}$ minute. ${ }^{7,8}$

A statistical test was done using MedCalc version 17.9.7. Also, observing the effect of high leukocytes $>50000 / \mu \mathrm{L}$ and the influence of hematocrit on ESR measurement was done using Starrsed RS. The Ethics Committee had approved this study, number 24/UN2.F1/ETIK/2017.

\section{RESULTS AND DISCUSSION}

This comparative study included a total of 47 subjects of the 1-20 mm ESR group, 52 subjects in the 21-60 mm ESR group, and 41 subjects in the $>60$ $\mathrm{mm}$ ESR group. The number of the samples met the criteria of ICSH, which specified a sample should include at least 40 subjects for each group. The range of values assessed in the sample comprised the 2-120 $\mathrm{mm}$ range recommended by $\mathrm{ICSH}^{1}{ }^{1}$ Table 1 show subject characteristics included in this study. Thirteen samples gave hazy readings on the instrument, consisting of five samples in the 60-minute mode, seven samples in the 30-minute mode, and one sample in the 30 and 60-minute modes.

Table 1. Subject characteristics

\begin{tabular}{lc}
\hline Sex & Total $(\mathbf{n}=\mathbf{1 4 0})$ \\
\hline Males & $41(29.3 \%)$ \\
Females & $99(70.1 \%)$ \\
Age (Median, in year) & $44.5(1-82)$ \\
Mean Hb (g/dL) & $11.96(1.99)$ \\
Mean Ht (\%) & $36.06(5.63)$ \\
Median of Leukocyte $\left(\times 10^{3} / \mathrm{uL}\right)$ & $7.22(2.62-80.67)$ \\
\hline
\end{tabular}

Spearman correlation test between manual ESR and Starrsed RS 60-minute mode showed a solid correlation with $r=0.98$ (95\% CI 0.97 to 0.98 ) and $p$ of $<0.0001$. Bland-Altman showed a mean bias of -0.4 $\mathrm{mm}(95 \% \mathrm{CI}-1.46$ to 0.74$)$ and was obtained with a $95 \%$ limit of agreement -13.25 to 12.54 . Ten (7.1\%) data were out of the limit of agreement, so there was a $92.9 \%$ of data which were still included in the $95 \%$ limit of agreement (Figure 1).

This results did not meet the ICSH requirement, recommending a 95\% difference between the two methods not more than $5 \mathrm{~mm}^{1}$ Nevertheless, the mean bias in this study was smaller than the mean bias for Ves-Matic Cube 200 and iSED obtained the Bogdaycioglu et al. study. ${ }^{9}$ Of the ten outliers, two samples gave hazy readings $<25$ in the 60 -minute mode. Unknown causes of other samples were out of the $95 \%$ limit of agreement. A total of $92.9 \%$ data were still included in the $95 \%$ limit of agreement and met the acceptable minimum limit for a sample size of 140 subjects which was $91 \%$ data even within 95\%limit of agreement. ${ }^{10}$

The result of the Passing-Bablok regression test (Figure 2) obtained a Cusum test for linearity of $p=0.07$. Thus there was a linear correlation between the two methods. Also, the Passing-Bablok regression test could be applied. The results of the intercept confidence interval were obtained at -1.00 (95\% CI -1.50 to 0.36 ) and slope of 1.00 (95\% CI 0.96 


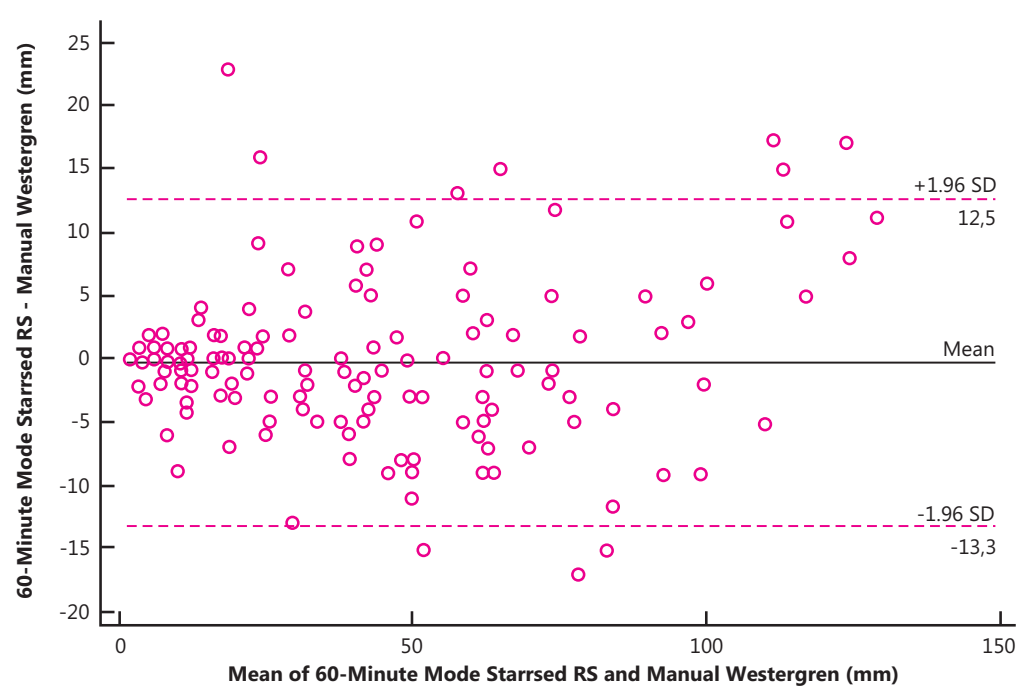

Figure 1. Bland-Altman plot of ESR results measured by manual Westergren method and 60-minute mode Starrsed RS

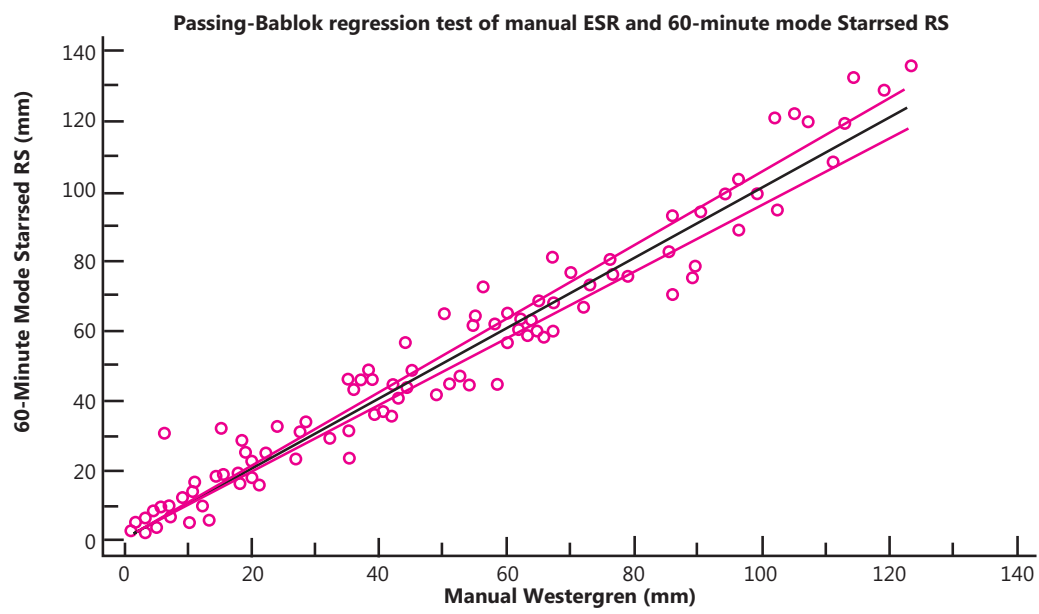

Figure 2. Passing-Bablok regression analysis of ESR results measured by manual Westergren method and 60-minute mode Starrsed RS

to 1.03). The confidence interval slope passed through 1 . Thus there was no proportional difference on both methods. Intercept confidence interval passing through 0 indicated no systematic difference in both methods. Residual Standard Deviation (RSD) in the ratio of both methods was 4.69 (95\% CI -9.19 to 9.19). This RSD value showed a random difference in the two comparable methods. No previous study had analyzed the comparison of the Starrsed RS with 60-minute mode and the Westergren manual method.

The results of Spearman correlation test between manual ESR and Starrsed RS 30-minute modes proved solid correlation with $r=0.97$ (95\% CI 0.96 to 0.98 ), and this was statistically significant $(p<0.0001)$. This research was similar to the previous study from Curvers et al. who obtained very strong correlation coefficient results $[r=0.96$ ( $95 \% \mathrm{CI} 0.93$ to $0.98)$. $^{2}$ A previous study by Rogers showed a correlation of 0.984 between Starrsed 30-minute mode with the 60-minute mode. ${ }^{5}$ Besides, this was similar to Berzelius et al. who applied a modified Sedimatic 100 ESR instrument using 30-minute sedimentation $(r=0.982){ }^{5}$ The correlation between the two Starrsed RS instrument modes measured by Westergren manually was better than the previous study. The previous study on Ves-Matic Cube 200 instrument read ESR within 20 minutes $r=0.83$ (95\% CI 0.76-0.88)] and an iSED instrument read ESR within 20 seconds $(r=0.76){ }^{2}$

Based on the Bland-Altman analysis (Figure 3), the mean bias was $-2.9 \mathrm{~mm}$ (95\% CI -4.23 to -1.58$)$ with a $95 \%$ limit of agreement -18.40 to 12.59 . This result was different from Horsti et al. study which 
compared the Starrsed Autocompact instrument with the Westergren method and found a mean of $4.1 \mathrm{~mm}$ bias with a $95 \%$ limit of agreement -24 to 33. ${ }^{11}$ Starrsed Autocompact instrument applied Westergren method modification and sedimentation assessment in 30 minutes. ${ }^{11}$

There were nine (6.4\%) data which were out of the $95 \%$ limit of agreement, so there were $93.6 \%$ of data within the $95 \%$ limit of the agreement which was still the acceptable minimum limit for a sample size of 140 subjects which was a $91 \%$ data still within 95\% limit of the agreement. ${ }^{10}$ Seven data had high ESR values. This result indicated that the possibility of reading in the 30-minute mode was not accurate for high ESR. Four samples were known to give a hazy reading in 30-minute mode. One sample had a high leukocyte count $(80670 / \mu \mathrm{L})$. The presence of three phases on the nonlinear sedimentation process became the major cause of the results of the study. The rate of sedimentation occurred through three phases, the formation of rouleaux/lag stage-rouleaux formation (0-20 minute), sedimentation/ decantation stage-sedimentation (15-30 minute), cell compaction/packing stage (25-60 minute). Starrsed RS 30-minute mode assessed the sedimentation rate of blood in the $30^{\text {th }}$ minute then using a prediction model to estimate the rate of sedimentation of blood in the $60^{\text {th }}$ minute.

In the $30^{\text {th }}$ minute, the new erythrocytes completed the sedimentation phase, while the new solidification phase was started. ${ }^{3}$ The occurrences found in this study were similar to the previous study, which employed Starrsed Autocompact instrument assessing ESR within 30 minutes. In that study, it was mentioned that the 30-minute mode might be too short for some samples and might cause errors, especially in high ESR, although the correlation was still acceptable. The use of 30-minutes mode might cause an ESR with 60-minute overestimation. In Kallner's study, it was found that $3 \%$ of the samples had faster sedimentation and reached the end of the reaction earlier than 60 minutes. ${ }^{12}$ In the sample under these conditions, the use of a 30-minute ESR measurement could yield significantly higher results than the actual one. ${ }^{12}$ These explained the cause of the outlier likely to be at a high ESR (7 out of 9 outliers) and observation of this outlier showed trends towards overestimation. Two data which were out of the limit of agreement in the ESR group of 21-60 mm were undetectable for the cause of the 95\% limit of agreement.

The result of the Passing-Bablok regression test (Figure 4) obtained a Cusum test for linearity $p=0.34$. Thus there was a linear correlation between the two methods. Therefore, Passing-Bablok regression test could be applied. The results of the Passing-Bablok regression test obtained a slope of 0.96 (95\% CI 0.93 to 1 ) and an intercept of -2.67 (95\% CI -4.00 to -1.72$)$. The slope confidence interval passed through 1 . Thus there was no proportional difference on both methods. The intercept confidence interval did not exceed the number 0 , indicating a systematic difference in both methods. The residual standard deviation in the ratio of both methods was 5.93 (95\% CI -11.64 to 11.64). This result was different from the study conducted by Curvers et al. who found a slope of 1.22 (95\% CI 1.11 to 1.33 ) and an intercept 3.14 (1.17 to 6.61) which applied 30-minute mode. ${ }^{2}$

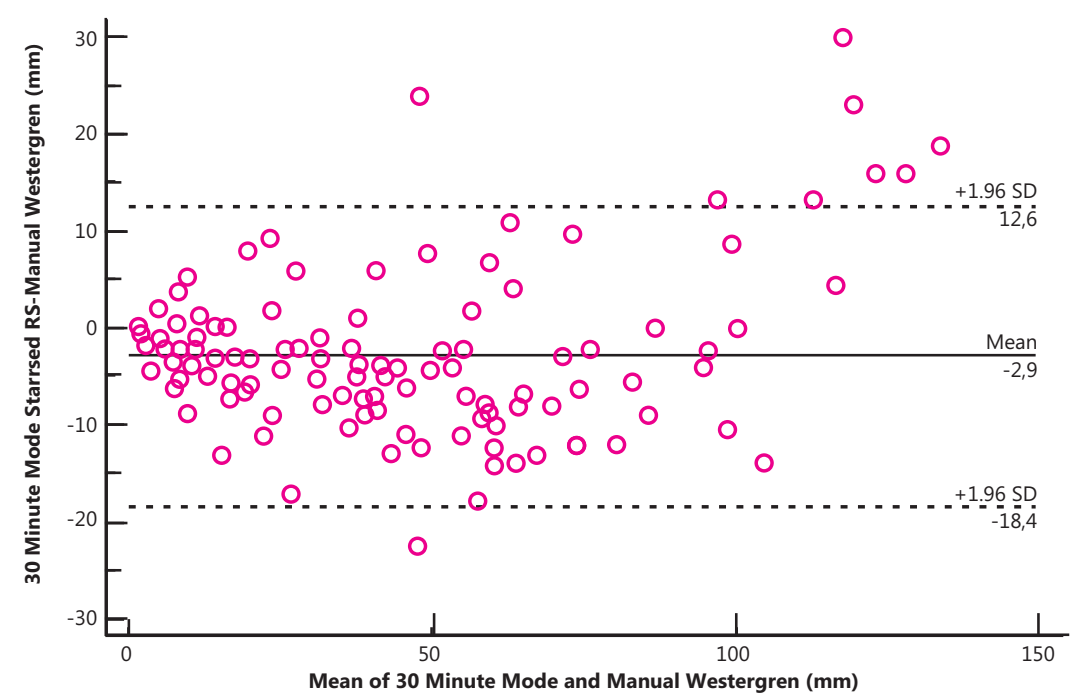

Figure 3. Bland-Altman plot of ESR results measured by manual Westergren method and 30-minute mode Starrsed RS 
This study used the Starrsed RS 30-minute mode after 60-minute mode was completed due to limitations of sample volume. Also, the instrument could only be run in one uniform mode for all samples, so there was quite a long interval between blood sampling and the Starrsed RS 30-minute mode. According to CLSI, the test can still be done within four hours after the sample is taken. ${ }^{4}$ This was also following the Bogdaycioglu et al. study which analyzed the stability of the sample on a Ves-Matic Cube 200 instrument using a modified Westergren principle with a reading of 20 minutes. ${ }^{9}$

From the Wilcoxon test, there was no significant difference between the manual ESR and Starrsed 60-minute mode, but there were significant differences between the manual ESR and 30-minute

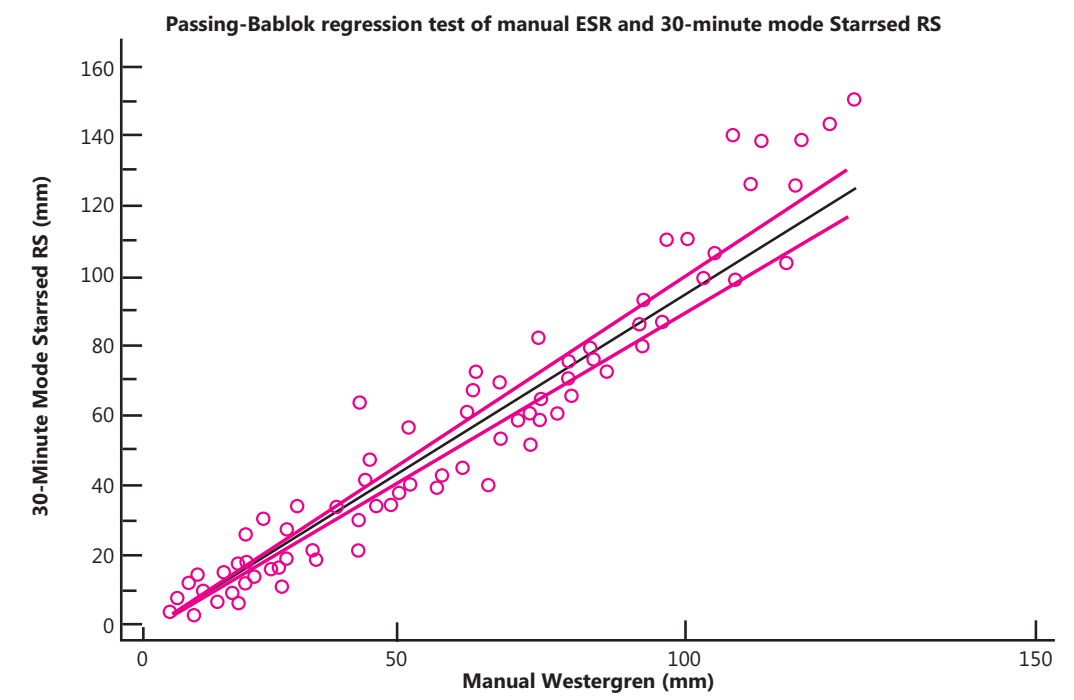

Figure 4. Passing-Bablok regression analysis of ESR results measured by manual Westergren method and 30-minute mode Starrsed RS

Table 2. Erythrocyte sedimentation rate results in samples with high leukocyte counts

\begin{tabular}{|c|c|c|c|c|c|}
\hline No & $\begin{array}{c}\text { Leukocyte } \\
\left(\times 10^{3} / \mu \mathrm{L}\right)\end{array}$ & $\begin{array}{l}\text { Starrsed RS } \\
60 \text {-minute } \\
\text { mode }\end{array}$ & $\begin{array}{c}\text { Manual } \\
\text { Westergren } \\
(\mathbf{m m})\end{array}$ & Info & $\begin{array}{l}\text { The difference of } \\
\text { Starrsed RS } \\
\text { Manual Method }\end{array}$ \\
\hline 1 & 252.12 & 133 & 125 & Hazy $>25$ & 8 \\
\hline 2 & 211.4 & 1 & 7 & & -6 \\
\hline 3 & 204.59 & 75 & 92 & Hazy $>25$ & -17 \\
\hline 4 & 190.21 & 3 & 12 & & -9 \\
\hline 5 & 180.19 & 2 & 16 & & -14 \\
\hline 6 & 138.23 & 2 & 13 & & -11 \\
\hline 7 & 131.51 & 46 & 57 & Hazy $>25$ & -11 \\
\hline 8 & 123.01 & 51 & 55 & Hazy $>25$ & -4 \\
\hline 9 & 120.82 & 42 & 43 & Hazy $>25$ & -1 \\
\hline 10 & 111.39 & 48 & 59 & Hazy $>25$ & -11 \\
\hline 11 & 110.71 & 57 & 76 & Hazy $>25$ & -19 \\
\hline 12 & 107.88 & 21 & 23 & Hazy $<25$ & -2 \\
\hline 13 & 88.45 & 45 & 37 & Hazy $>25$ & 8 \\
\hline 14 & 80.67 & 135 & 124 & & 11 \\
\hline 15 & 76.71 & 110 & 115 & & -5 \\
\hline 16 & 73.63 & 12 & 16 & Hazy $<25$ & -4 \\
\hline 17 & 68.82 & 38 & 46 & Hazy $<10$ & -8 \\
\hline 18 & 68.46 & 68 & 66 & Hazy $>25$ & 2 \\
\hline 19 & 66.54 & 78 & 77 & Hazy $<10$ & 1 \\
\hline 20 & 65.97 & 48 & 51 & Hazy $>25$ & -3 \\
\hline 21 & 63.28 & 38 & 37 & Hazy $>25$ & 1 \\
\hline
\end{tabular}


Table 3. Comparison of sample result with $\mathrm{Ht} \leq 35 \%$ and $\mathrm{Ht}>35 \%$

\begin{tabular}{cccccc}
\hline & Hematocrit & N & $\begin{array}{c}\text { Mean manual } \\
\text { ESR }(\mathbf{m m})\end{array}$ & $\begin{array}{c}\text { Mean Starrsed } \\
\text { RS 60 min } \\
\text { mode (mm) }\end{array}$ & $\mathbf{p}$ \\
\hline ESR 1-20 $\mathbf{~ m m}$ & $\leq 35 \%$ & 14 & $13(4.80)$ & $13.4(7.86)$ & 0.7719 \\
& $>35 \%$ & 32 & $10.3(4.84)$ & $10(2-30)$ & 0.7879 \\
ESR 21-60 $\mathbf{~ m m}$ & $\leq 35 \%$ & 16 & $42.9(12.68)$ & $44.2(15.10)$ & 0.4478 \\
& $>35 \%$ & 37 & $38.2(11.05)$ & $36.4(11.44)$ & 0.0980 \\
ESR>60 $\mathbf{~ m m}$ & $\leq 35 \%$ & 28 & $86.5(61-124)$ & $79.5(56-135)$ & 0.7759 \\
& $>35 \%$ & 13 & $68(63-108)$ & $68(58-119)$ & 0.2734 \\
\hline
\end{tabular}

mode. This result was inconsistent with the previous study by Emelike et al. ${ }^{13}$ Thus, this study confirmed that the ESR reading should be done in 60 minutes. ${ }^{14}$

Twenty-one samples with high leukocytes were found in this study (Table 2). Fifteen samples showed hazy results on Starrsed RS 60-minute mode, consisting of 11 samples resulting in hazy readings $>$ $25 \mathrm{~mm}, 2$ hazy sample $<25 \mathrm{~mm}$, and 2 hazy samples $<10 \mathrm{~mm}$. Saphiro-Wilk statistical test showed that the data distribution of the ESR test in both methods was abnormal. In the Wilcoxon test, there were significant differences between the manual method and Starrsed 60-minute mode in samples with high leukocyte $(p=0.0208)$.

There was no data from previous studies which had searched for leukocyte effect on Starrsed RS readings. The possible cause of this different reading was the blurred boundary between erythrocytes and plasma. However, according to CLSI, the cause of this blurred was still unclear. According to Osei-Bimpong et al. ${ }^{6}$ It was mentioned that reticulocytes caused the blurred boundary between erythrocytes and plasma, however, in this study, no data were collected on reticulocyte counts in the sample. ${ }^{6}$ In five samples that did not result in hazy readings, the ESR read by the instrument was lower than those read by the manual method. This result was probably due to the thick buffy coat in the sample of high leukocytes read by the instrument as an erythrocyte column and the instrument automatically read the erythrocyte boundary with the plasma based on the percentage of light absorption.

The effects of hematocrit on ESR measurement by Starrsed RS 60-minute mode can be seen in Table 3.

The result of a statistical test showed no significant difference in ESR reading in 60-minute mode and manual method in the sample with $\mathrm{Ht} \leq 35 \%$ and $>35 \%$. This result was consistent with the instrument performance, which claimed that the readings in Starrsed RS were not affected by the hematocrit. Changes in plasma viscosity and hematocrit may cause clogging of the Westergren pipette by quickly decreasing erythrocyte aggregates. ${ }^{3,15,16}$ Samples with a high hematocrit (> 35\%) and a high ESR affect the ESR since it can form a short, randomized block effective tube length causing slow ESR. To overcome this, CLSI recommended using a diameter of the pipette, which is not less than $2.55 \mathrm{~mm}$. The Starrsed RS instrument used a similar pipette to ESR test measured by the Westergren manual method. ${ }^{4,16}$ The sample dilution was expected to lead to measurements free of viscosity and hematocrit difference. ${ }^{1}$

\section{CONCLUSION AND SUGGESTION}

Erythrocyte sedimentation rate test measured by Starrsed RS 60-minute mode correlated very strongly, had appropriateness and generated no significant difference with the manual Westergren ESR method. Erythrocyte sedimentation rate measured by Starrsed RS 30-minute mode had a very strong correlation, and had appropriateness to the manual method. There were systematic and significant differences in the average test results. Researchers recommend that ESR at the Starrsed RS be read at 60 minutes mode. The leukocyte count of $>50,000 /$ uL might affect ESR reading with the Starrsed RS automated instrument, whereas the hematocrit level did not affect the ESR reading on the instrument. ${ }^{4}$

\section{ACKNOWLEDGEMENT}

Researchers would like to thank Andi Mulyawan for his assistance in this research. We would also like to thank PT Saba Indomedika for providing equipment and reagents for this research.

\section{REFERENCES}

1. Jou JM, Lewis SM, Briggs C, Lee SH, Salle B, McFadden $\mathrm{S}$. ICSH review of the measurement of the erythrocyte 
sedimentation rate. Int JnI Lab Hem 2011; 33: 125-32.

2. Curvers J, Kooren J, Laan M, Lierop E, Kerkhof D, Scarnhorst V, et al. Evaluation of the Ves-Matic Cube 200 erythrocyte sedimentation method: Comparison with Westergren-based method. Am J Clin Pathol 2010; 134: 653-60.

3. RR Mechatronics. A classic gold standard: The Westergren method for ESR measurement [Internet]. Netherlands: RR Mechatronics; c2016. [updated 2016 Feb; cited 2016 Nov 30]. Available from: www.rrmechatronics.com.

4. Clinical and Laboratory Standards Institute. Procedures for erythrocyte sedimentation rate test; approved standard. Fifth Ed., CLSI document H02-A5. Wayne: The Clinical and Laboratory Standards Institute, 2011; 1-14.

5. Rogers R. Erythrocyte sedimentation rate: 30 minute values [Internet]. Netherlands, Medical Laboratory Worlds; c2017 [updated 1993; cited 2016 Nov 30]. Available from: https://rrmechatronics.com/ esr/publications/.

6. Osei-Bimpong A, Burthem J. Supplementary techniques including blood parasite diagnosis. In: Bain BJ, Bates I, Laffan MA, Lewis SM, editors. Dacie and Lewis Practical Haematology. $11^{\text {th }}$ Ed., China, Elsevier, 2011; 102-5.

7. RR Mechatronics. Starred RS User Manual. Netherland, RR Mechatronics International B.V. 2015.

8. Manley RW. The effect of room temperature on erythrocyte sedimentation rate and its correction. J Clin Path 1957; 10: 354

9. Bogdaycioglu N, Yilmaz FM, Sezer S, Oguz E. Comparison of iSED and Ves-Matic Cube 200 erythrocyte sedimentation rate measurements with
Westergren method. J Clin Lab Anal 2014; 00: 1-6.

10. Linnet K, Boyd JC. Selection and analytical evaluation of methods with statistical techniques. In: Burtis CA, Ashwood ER, Bruns DE, editors. Tietz textbook of clinical chemistry and molecular diagnostics. $5^{\text {th }}$ Ed., Missouri, Elsevier, 2012; 7-48.

11. Horsti J, Rontu R, Collings A. A comparison between the Starrsed Auto-Compact erythrocyte sedimentation rate instrument and the Westergren method. J Clin Med Res 2010; 2(6): 261-5.

12. Kallner A, Engervall P, Bjorkholm M. Kinetic measurement of the erythrocyte sedimentation rate. Upsala J Med Sci, 1994; 99: 179-86.

13. Emelike OF, Akpan JE, Obigwe BU, Jeremiah ZA. Comparative study of Erythrocytes Sedimentation Rate (ESR) using trisodium citrate, normal saline, and whole blood in Ethylene Diamine Tetra Acetic Acid (EDTA). J Appl Sci Environ Manage, 2010; 14 (1): 23-7.

14. College of Physicians and Surgeons of Alberta. Erythrocyte sedimentation rate [Internet]. Alberta: Alberta Laboratory Quality Enhancement Program. c2004 [updated 2000 May; cited 2017 Mar 24]. Available from: https://www.cpsa.ca/wpcontent/uploads/2016/08/ESR-EducationalDocument-.pdf/.

15. International Council for Standardization in Haematology. ICSH recommendations for measurement of erythrocyte sedimentation rate. J Clin Pathol, 1993; 46: 198-203.

16. Clinical and Laboratory Standards Institute. Reference and selected procedures for erythrocyte sedimentation rate test; approved standard. Fourth Ed., CLSI document H02-A4. Wayne: The Clinical and Laboratory Standards Institute. 2000; 1-14. 